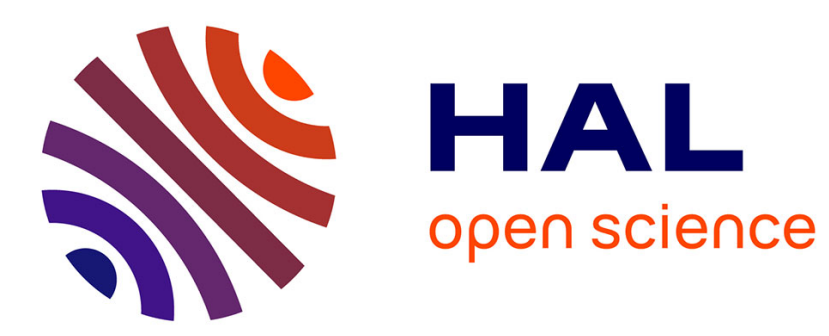

\title{
Single dataset methods and deterministic-aided STAP for heterogeneous environments
}

Jean François Degurse, Laurent Savy, Sylvie Marcos

\section{To cite this version:}

Jean François Degurse, Laurent Savy, Sylvie Marcos. Single dataset methods and deterministic-aided STAP for heterogeneous environments. 2014 International Radar Conference, Oct 2014, Lille, France. hal-01102174

\section{HAL Id: hal-01102174 \\ https://hal-centralesupelec.archives-ouvertes.fr/hal-01102174}

Submitted on 2 Jul 2020

HAL is a multi-disciplinary open access archive for the deposit and dissemination of scientific research documents, whether they are published or not. The documents may come from teaching and research institutions in France or abroad, or from public or private research centers.
L'archive ouverte pluridisciplinaire HAL, est destinée au dépôt et à la diffusion de documents scientifiques de niveau recherche, publiés ou non, émanant des établissements d'enseignement et de recherche français ou étrangers, des laboratoires publics ou privés. 


\section{Single dataset methods and deterministic-aided STAP for heterogeneous environments}

\author{
Jean-François Degurse \\ Thales Airborne Systems \\ 2, Avenue Gay Lussac \\ 78851 Elancourt, France \\ Email: jean-francois.degurse@fr.thalesgroup.fr
}

\author{
Laurent Savy \\ Sylvie Marcos \\ Electromagnetism and Radar Dept. Laboratoire des Signaux et des Systèmes \\ ONERA \\ Palaiseau, France \\ Email: laurent.savy@onera.fr
}

\begin{abstract}
Classical space-time adaptive processing (STAP) detectors are strongly limited when facing highly heterogeneous environments. Indeed, in this case, representative target free data are no longer available. Single dataset algorithms such as the MLED algorithm, have proved their efficiency in overcoming this problem by only working on primary data. These methods are based on the APES algorithm which removes the useful signal from the covariance matrix. However, a small part of the clutter signal is also removed from the covariance matrix in this operation. Consequently a degradation of clutter rejection performance is observed. We propose two algorithms that use deterministic-aided STAP to overcome this issue of the single dataset APES method. The results on realistic simulated data and real data show that these methods outperform traditional single dataset methods in detection and in clutter rejection.
\end{abstract}

\section{INTRODUCTION}

In the context of radar signal processing, the purpose of Space-time adaptive processing (STAP) is to remove ground clutter returns, in order to enhance slow moving target detection. STAP performs two-dimensional space and time adaptive filtering where different space channels are combined at different times [1]. Filter's weights are adaptively computed from training data in the neighbourhood of the range cell of interest, called cell under test (CUT). The estimation of these weights is always deducted, more or less directly, from an estimation of the covariance matrix of the received signal, which is the key quantity in the process of adaptation [2]. Any implementation of STAP processing must remain absolutely consistent with the strategy of radar processing which purpose is to obtain a high probability of detection while keeping a very low probability of false alarm.

Classical space-time adaptive processing (STAP) detectors are strongly limited when facing a severe non stationary environment such as heterogeneous clutter. Indeed, in these cases, representative training data are no longer available. The Maximum Likelihood Estimation Detector (MLED) [3] is a single dataset detector among others [4]. It only operates with the data from the cell under test hence its performance is not impacted by nonstationarity. Of course, no environment is purely heterogeneous or homogeneous and the problem can be addressed by combining primary and secondary data [5]. We will here consider the environment to be heterogeneous enough to only use primary data. To make the primary data target-free, the MLED detector removes a thin part of the signal of the Doppler cell under test from the covariance matrix. A slight part of the clutter is removed along the target signal which implies a degradation of clutter rejection, especially if the number of Doppler cells is low. Less there are Doppler cells, more clutter is removed from the covariance matrix and worse the estimation of the covariance matrix is. The bad estimation of the matrix can be addressed by using subspace methods [6] but the removal of some clutter is inherent to the APES method.

In this paper, we will show how we can overcome this problem by the use of deterministic-aided STAP. Moreover, we will extend this method to the Stop-Band APES which greatly reduces the computational workload of the MLED detector.

Section II is devoted to the data model, and section III summarizes the principle of the MLED APES-based detector and the Stop-Band APES algorithm. A deterministic based non-adaptive approach of space-time processing is presented in section IV. In section V, we describe two different approach for deterministic-aided STAP and finally in section VI, simulations are given to show that the proposed methods outperform the MLED and Stop-Band algorithms.

\section{DATA MODEL}

Consider a radar antenna made of $N$ sensors that acquires $M_{p}$ pulse snapshots for each range gate $l$. We will only use the primary data so we will forget the range gate dimension, also called fast-time dimension. Then the processing algorithm works independently in each range cell. We adopt the following two hypothesis model where $H_{0}$ and $H_{1}$ means that no target or a target is present, respectively :

$$
\begin{gathered}
H_{0}: \mathbf{X}=\mathbf{N} \\
H_{1}: \mathbf{X}=\alpha \mathbf{s}_{\mathbf{s}} \mathbf{s}_{\mathbf{t}}{ }^{\mathbf{T}}+\mathbf{N}
\end{gathered}
$$

where the received data have been arranged into an $M N \times K_{t}$ matrix $\mathbf{X}$ with $K_{t}$ being the number of training data pulse snapshots, $M$ the number of pulses of the spatio-temporal vector, $\alpha$ the complex amplitude of the target. $\mathbf{s}_{\mathbf{s}}$ is the spatio-temporal steering vector (length $N M$ ), $\mathbf{s}_{\mathbf{t}}$ the temporal steering vector (length $K_{t}=M_{p}-M+1$ ) and $\mathbf{N}$ is the interference (clutter plus noise) matrix.

We make use of a temporal sliding window to work on the temporal dimension, consequently the estimated covariance 
matrix $\mathbf{R}$ is obtained from $\mathbf{X}$ as follows :

$$
\mathbf{R}=\frac{1}{K_{t}} \mathbf{X X}^{\mathbf{H}}
$$

One classical STAP detector taken as reference uses the Adaptive Matched Filter (AMF) [1] [2]. The filter $\mathbf{w}$ is:

$$
\mathbf{w}=\frac{\mathbf{R}^{-1} \mathbf{s}}{\mathbf{s}_{\mathbf{s}}^{\mathbf{H}} \mathbf{R}^{-1} \mathbf{s}_{\mathbf{s}}}
$$

Detection is achieved comparing the output SNIR power of the matched filter to a threshold:

$$
\mathbf{P}_{A M F}=\frac{\left|\mathbf{s}_{\mathbf{s}}^{\mathbf{H}} \mathbf{R}^{-1} \mathbf{X}\right|^{2}}{\mathbf{s}_{\mathbf{s}}^{\mathbf{H}} \mathbf{R}^{-1} \mathbf{s}_{\mathbf{s}}} \underset{H_{1}}{\stackrel{H_{0}}{\lessgtr}} \eta
$$

In case where a strong target is present at this range gate, $\mathbf{R}$ contains the target covariance matrix. Consequently, the target is removed with the clutter and it can no longer be detected by (5). This happens when many targets are moving at the same speed but are at different distances (roads, highways, convoys...). Another problem with this detector is that the ground clutter has to be homogeneous on the range domain. Otherwise the clutter used to estimate the covariance matrix won't be representative of the clutter that has to be canceled, leading to a bad clutter rejection.

\section{APES-BASED STAP DETECTORS}

\section{A. The Maximum Likelihood Estimation Detector}

To overcome the previous issues of signal suppression or none representativeness of secondary data, the MLED detector [7] based on the APES [8] algorithm removes the signal of interest from the covariance matrix. The problem is stated as follows:

$$
\min _{w, \alpha}\left(\mathbf{w}^{\mathbf{H}} \mathbf{X}-\alpha \mathbf{s}_{\mathbf{t}}^{\mathbf{T}}\right)\left(\mathbf{w}^{\mathbf{H}} \mathbf{X}-\alpha \mathbf{s}_{\mathbf{t}}^{\mathbf{T}}\right)^{H} \text { s.t } \mathbf{w}^{\mathbf{H}} \mathbf{S}_{\mathbf{s}}=1
$$

The obtained solution is :

$$
\mathbf{w}=\frac{\mathbf{Q}^{-1} \mathbf{s}_{\mathbf{s}}}{\mathbf{s}_{\mathbf{s}}^{\mathbf{H}} \mathbf{Q}^{-1} \mathbf{S}_{\mathbf{s}}} \text { and } \alpha=\frac{\mathbf{w}^{\mathbf{H}} \mathbf{X} \mathbf{s}_{\mathbf{t}}^{*}}{K_{t}}
$$

where

$$
\mathrm{Q}=\mathbf{R}-\mathrm{gg}^{\mathbf{H}}, \mathbf{g}=\frac{\mathbf{X} \mathbf{s}_{\mathbf{t}}^{*}}{\mathbf{K}_{\mathbf{t}}}
$$

Detection is achieved using the output power normalized by the Adaptive Power Residue (APR $=\mathbf{w}^{\mathbf{H}} \mathbf{Q w}=\mathbf{s}_{\mathbf{s}}^{\mathbf{H}} \mathbf{Q}^{-1} \mathbf{S}_{\mathbf{s}}$ ):

$$
\mathbf{P}_{M L E D}=\frac{\left|\mathbf{s}_{\mathbf{s}}^{\mathbf{H}} \mathbf{Q}^{-1} \mathbf{g}\right|^{2}}{\mathbf{s}_{\mathbf{s}}^{\mathbf{H}} \mathbf{Q}^{-1} \mathbf{s}_{\mathbf{s}}} \underset{H_{1}}{\stackrel{H_{0}}{\lessgtr}} \eta
$$

To avoid strong signal loss due to covariance matrix estimation erros [9], one may uses in addition diagonal loading [10] or subspace methods [11].

\section{B. Extension to Stop-Band APES}

Because the MLED algorithm is a high resolution method, it requires an oversampling in Doppler frequency, typically by a factor four, to correctly work. Indeed, combining (6) and (7), it follows:

$$
\begin{gathered}
\mathbf{w}^{\mathbf{H}}\left(\mathbf{X}-\mathbf{X} \frac{\mathbf{s}_{\mathbf{t}}^{*} \mathbf{s}_{\mathbf{t}}^{\mathbf{T}}}{\mathbf{s}_{\mathbf{t}}^{\mathbf{T}} \mathbf{s}_{\mathbf{t}}^{*}}\right)\left(\mathbf{X}-\mathbf{X} \frac{\mathbf{s}_{\mathbf{t}}^{*} \mathbf{s}_{\mathbf{t}}^{\mathbf{T}}}{\mathbf{s}_{\mathbf{t}}^{\mathbf{T}} \mathbf{s}_{\mathbf{t}}^{*}}\right)^{H} \mathbf{w} \\
=\mathbf{w}^{H} \mathbf{X}\left(\mathbf{I}-\mathbf{P}_{/ /}\right)\left(\mathbf{I}-\mathbf{P}_{/ /}\right)^{H} \mathbf{X} \mathbf{w}
\end{gathered}
$$

where $\mathbf{P}_{/ /}$is the projector into the target signal subspace:

$$
\mathbf{P}_{/ /}=\frac{\mathbf{s}_{\mathrm{t}}^{*} \mathbf{s}_{\mathbf{t}}^{\mathbf{T}}}{\mathbf{s}_{\mathbf{t}}^{\mathbf{T}} \mathbf{s}_{\mathbf{t}}^{*}}=\frac{\mathbf{s}_{\mathbf{t}}^{*} \mathbf{s}_{\mathbf{t}}^{\mathbf{T}}}{K_{t}}
$$

The problem (6) can then be recognized as a minimization of the interference plus noise energy outside the subspace spanned by the target:

$$
\min _{\mathbf{w}}\left\{\mathbf{w}^{\mathbf{H}} \mathbf{X}\left(\mathbf{I}-\mathbf{P}_{/ /}\right)\left(\mathbf{I}-\mathbf{P}_{/ /}\right)^{H} \mathbf{X} \mathbf{w}\right\} \text { s.t } \mathbf{w}^{H} \mathbf{s}_{s}=1
$$

The solution is still $\mathbf{w}=\frac{\mathbf{Q}^{-1} \mathbf{s}_{\mathrm{s}}}{\mathbf{s}_{\mathrm{s}}^{\mathrm{H}} \mathbf{Q}^{-1} \mathbf{s}_{\mathrm{s}}}$, but with the more general form for $\mathbf{Q}$ :

$$
\mathbf{Q}=\frac{\mathbf{X X}^{H}}{\mathbf{s}_{\mathbf{t}}^{\mathbf{T}} \mathbf{s}_{\mathbf{t}}^{*}}-\frac{1}{K_{t}} \mathbf{X P} / / \mathbf{X}^{H}
$$

This latest formulation not only shows the hyper resolution property along the frequency domain, but also allows to overcome one major drawback of the MLED method for our application. The MLED has indeed a high frequency resolution due to the sharpness of the projection $\mathbf{I}-\mathbf{P}_{/ /}$with $\mathbf{P}_{/ /}=\frac{\mathbf{s}_{t}^{*} \mathbf{s}_{t}^{\mathrm{T}}}{\mathbf{s}_{\mathbf{t}}^{\mathrm{T}} \mathbf{s}_{\mathbf{t}}^{*}}$ (dash curve, Fig. 1). This is a problem because it requires a strong oversampling to be sure to remove the signal of interest from the covariance matrix, and so leads to an important increase of the computing load. In order to avoid this problem, we propose a new detector called Stop-Band APES. The minimization is using a projector $\mathbf{P}_{/ /}$on an extended subspace around the Doppler frequency $f_{0}$ under test. For instance, two adjacent half-cells can be added into the space spanned by $\mathbf{P}_{/ /}=\mathbf{S}_{t}^{*}\left(\mathbf{S}_{\mathbf{t}}^{\mathbf{T}} \mathbf{S}_{\mathbf{t}}^{*}\right)^{-1} \mathbf{S}_{\mathbf{t}}^{\mathbf{T}}$ with:

$$
\mathbf{S}_{t}=\left[\mathbf{s}_{\mathbf{t}}\left(f_{0}-\frac{1}{2 K_{t}}\right), \mathbf{s}_{\mathbf{t}}\left(f_{0}\right), \mathbf{s}_{\mathbf{t}}\left(f_{0}+\frac{1}{2 K_{t}}\right)\right]
$$

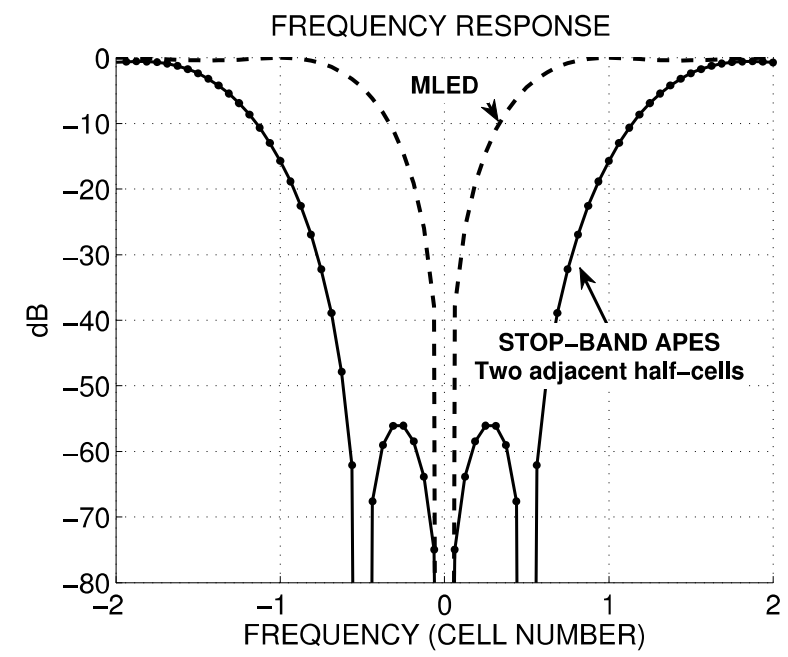

Fig. 1. Spectral response of regular MLED $S=s_{t}$ (dash curve) and StopBand APES with two adjacent half-cells (solid curve) 
The sharpness and effectiveness of the cancellation around the target signal are characterized by the frequency response of the projector, which is, for a signal $\mathbf{X}$ at frequency $f(\mathbf{X}=$ $\left.\mathbf{s}_{\mathbf{t}}^{\mathbf{T}}(f)\right)$ :

$$
\begin{aligned}
\tilde{P}_{\perp}(f)= & {\left[\mathbf{s}_{\mathbf{t}}^{\mathbf{T}}(f)\left(\mathbf{I d}-\mathbf{P}_{/ /}\right)\right] \frac{\mathbf{s}_{\mathbf{t}}^{*}(f)}{\mathbf{s}_{\mathbf{t}}^{\mathbf{T}}(f) \mathbf{s}_{\mathbf{t}}^{*}(f)} } \\
& =1-\mathbf{s}_{\mathbf{t}}^{\mathbf{T}}\left(f_{1}\right) \frac{\mathbf{P}_{/ /}}{K_{T}} \mathbf{s}_{\mathbf{t}}^{*}(f)
\end{aligned}
$$

Fig. 1 shows that building a projector with two adjacent halfcells is enough to correctly remove the signal in the cell under test. Nevertheless, compared to the MLED, the Stop-Band APES does not require oversampling of the Doppler resolution for the calculation and the application of the STAP filter. A zero-padding by a factor 2 will still be required to access the signal that has to be evaluated every half-resolution cells for the creation of the projector [12] [13].

\section{Limitations of the MLED and Stop-Band APES}

In order to explore the use of subspace-based methods, we have to go deeper in the formulation of the MLED detector. Indeed, these methods will only work if the clutter subspace of the covariance matrix $\mathbf{R}$ remains very close to the clutter subspace of the target free covariance matrix $\mathbf{Q}$. For a given distance cell, if there is no target at this range, the covariance matrix $\mathbf{R}$ only contains interference, i.e clutter and possibly jamming signal, and noise, according to (3) :

$$
\mathbf{R}=\frac{\mathbf{X X}^{\mathbf{H}}}{K_{t}}=\frac{\mathbf{N N}^{\mathbf{H}}}{K_{t}}
$$

We can demonstrate [11] than the matrix $\mathbf{Q}$ is, without approximation :

$$
\mathbf{Q}=\frac{\mathbf{N N}^{\mathbf{H}}}{K_{t}}-\frac{\mathbf{N s}_{\mathbf{t}}^{*} \mathbf{s}_{\mathbf{t}}^{\mathbf{T}} \mathbf{N}^{\mathbf{H}}}{K_{t}^{2}}
$$

The matrix $\frac{\mathrm{NN}^{\mathrm{H}}}{K_{t}}$ is the interference plus noise estimated covariance matrix whereas $\frac{\mathbf{N s}_{t}^{*} \mathbf{s}_{t}^{\mathrm{T}} \mathbf{N}^{\mathbf{H}}}{K_{t}^{2}}$ is the scalar product of interference plus noise vectors with their projection on $\mathbf{s}_{\mathbf{t}}^{*}$. It follows from (14) that the modified covariance matrix $\mathbf{Q}$ used for MLED in (9) does no longer contain the target contribution and that the target will not be removed contrarily to the clutter by the MLED STAP filter (7).

The residual clutter plus noise covariance matrix is slightly different from the actual covariance matrix $\frac{\mathbf{N N}^{\mathrm{H}}}{K_{t}}$ (Fig 2).The term $\frac{\mathrm{Ns}_{\mathrm{t}}^{*} \mathbf{s}_{t}^{\mathrm{T}} \mathbf{N}^{\mathrm{H}}}{K_{t}^{2}}$ represents the part of the clutter that is removed from the covariance matrix. The number of Doppler cells being usually high, the projector is consequently very sharp i.e the term $\frac{\mathbf{N s}_{t}^{*} \mathbf{s}_{t}^{\mathbf{T}} \mathbf{N}^{\mathbf{H}}}{K_{t}^{2}}$ is small and both MLED and Stop-Band APES, which removes a wider part of the clutter from the covariance matrix $\frac{\mathbf{N S}_{t}^{*} \mathbf{S}_{t}^{\mathbf{T}} \mathbf{N}^{\mathbf{H}}}{K_{t}^{2}}$, are all working. This effect can be seen on Fig. 2 in a situation with and without target in the Doppler cell tested.

However, in a situation where the number of Doppler cells is low, we will observe a degradation of the clutter rejection

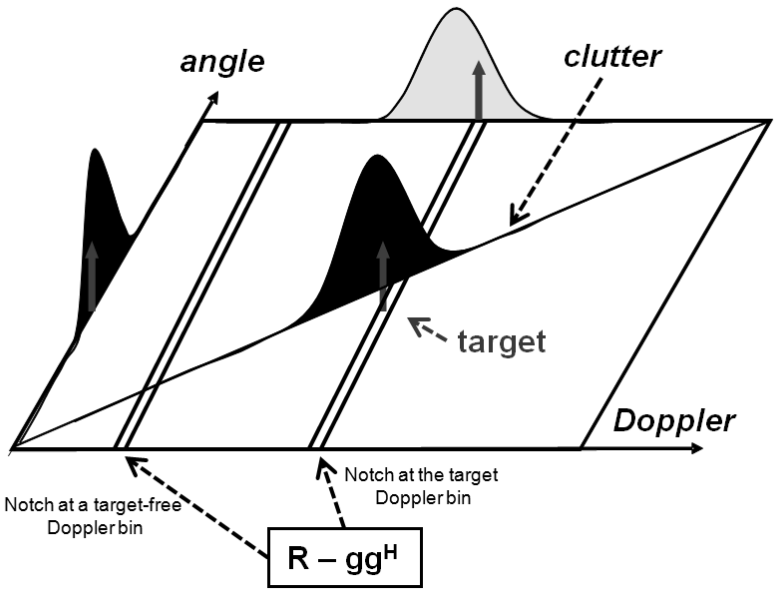

Fig. 2. Angle-Doppler map showing the effect of MLED projector for two different Doppler bins

performance of the MLED detector, and this degradation will be even worse for the Stop-Band APES algorithm. This effect is due to the partitioning which is done only in time domain. If spatio-temporal partitioning is employed, only a single bin of the angle-Doppler plane is removed but the computational cost would hugely increase because of the angle-Doppler scanning. We will present in the next section a deterministic processing and, in section $\mathrm{V}$, a new method that makes use of deterministic processing to solve this problem.

\section{DETERMINISTIC SPACE-TIME PROCESSING}

We will here briefly describe a non-adaptive space-time processing which is the basis of the deterministic-aided STAP processing we will introduce in the following section. For a side-mounted antenna, the clutter occupies a one dimension position in the two-dimensional Doppler-angle domain. The clutter Doppler frequency is a function of the receiving angle:

$$
f=\frac{2 V}{\lambda} \sin \theta \Rightarrow \theta=\sin ^{-1}\left(\frac{\lambda f}{2 V}\right)
$$

with $f$ the Doppler frequency of the clutter, $\theta$ the receiving angle, $V$ the platform speed and $\lambda$ the wavelength of the radar frequency. Knowing this relation, we can build a filter that will remove all the signal that is in the $1 \mathrm{D}$-domain driven by Eq. (15). The general form of the filter, which will be referred in the following to non-adaptive or deterministic processing, has the same form as AMF in (4):

$$
\mathbf{w}^{\mathbf{H}}=\frac{\mathbf{s}_{\mathbf{s}}^{\mathbf{H}} \mathbf{K}^{-\mathbf{1}}}{\mathbf{s}_{\mathbf{s}}^{\mathbf{H}} \mathbf{K}^{-1} \mathbf{s}_{\mathbf{s}}}
$$

but with

$$
\mathbf{K}=\frac{1}{k} \sum_{i=1}^{k} \mathbf{s}_{\mathbf{c}}\left(\theta_{i}\left(f_{i}\right), f_{i}\right) \mathbf{s}_{\mathbf{c}}^{\mathbf{H}}\left(\theta_{i}\left(f_{i}\right), f_{i}\right)+\boldsymbol{\Gamma}_{\mathbf{N}}
$$

where $\Gamma_{\mathbf{N}}$ is the true noise covariance matrix (identity matrix in our case), $k$ is the number of main lobe clutter patches, $\mathbf{s}_{\mathbf{c}}\left(\theta_{i}\left(f_{i}\right), f_{i}\right)$ is the space-time steering vector of angle $\theta_{i}$ and frequency $f_{i}$ obtained with (15). 
In the same formulation of the filter as MLED and StopBand APES in (7), the matrix for each Doppler cell can be written:

$$
\mathbf{K}^{\prime}=\frac{1}{k^{\prime}} \sum_{i=1}^{k^{\prime}} \mathbf{s}_{\mathbf{c}}\left(\theta_{i}\left(f_{i}\right), f_{i}\right) \mathbf{s}_{\mathbf{c}}^{\mathbf{H}}\left(\theta_{i}\left(f_{i}\right), f_{i}\right)+\boldsymbol{\Gamma}_{\mathbf{N}}
$$

the vector $\mathbf{s}_{\mathbf{c}}\left(\theta_{i}\left(f_{i}\right), f_{i}\right)$ is the predicted steering vector of the clutter. In this case, to process one Doppler cell, the steering vector $\mathbf{s}_{\mathbf{c}}\left(\theta_{0}\left(f_{0}\right), f_{0}\right)$ of the Doppler cell under test and the two steering vectors $\mathbf{s}_{\mathbf{c}}\left(\theta_{ \pm 1}\left(f_{ \pm 1}\right), f_{ \pm 1}\right)$ from the adjacent Doppler cells are sufficient to correctly remove the clutter. However, the performance of this non-adaptive approach is very limited in practical situations because of the heterogeneity of the clutter (e.g urban or mountainous areas) and because of antenna/receivers calibration errors which make the real steering vector of the antenna slightly different from the actual steering vector used to build the covariance matrix.

To illustrate this effect, we compare the non-adaptive processing (16) to the classic adaptive processing on realistic data. These data are simulated by a STAP simulator that emulates phases errors on the receiving channels and randomly adds impulsive echos in the clutter. Clutter is Gaussian, homogeneous and set at $40 \mathrm{~dB}$. No target are present in these data. A side looking antenna with four uniformly spaced sub-arrays is used. Aircraft speed is set at $100 \mathrm{~ms}^{-1}$, radar frequency is $10 \mathrm{GHz}$ and the pulse frequency (PRF) is $2 k H z$. The non-adaptive processing is only applied in the positive speed domain, that is to say that the negative speeds show the sum channel. The adaptive processing is applied on all the Doppler (speed) domain.

As we can see on Fig. 3 on the realistic simulated data, the non-adaptive processing is not performing well, as it fails to suppress the clutter. Indeed, the residual CNR is near $15 \mathrm{~dB}$ in the main lobe, the clutter attenuation is limited to $25 \mathrm{~dB}$, implying many false alarms. The full range-Doppler maps also points out this effect on Fig.4. From these results, we deduce that we cannot use a non-adaptive space-time processing in real situations but we may use the deterministic of the clutter Doppler-angle relation together with adaptive processing to achieve better performance.

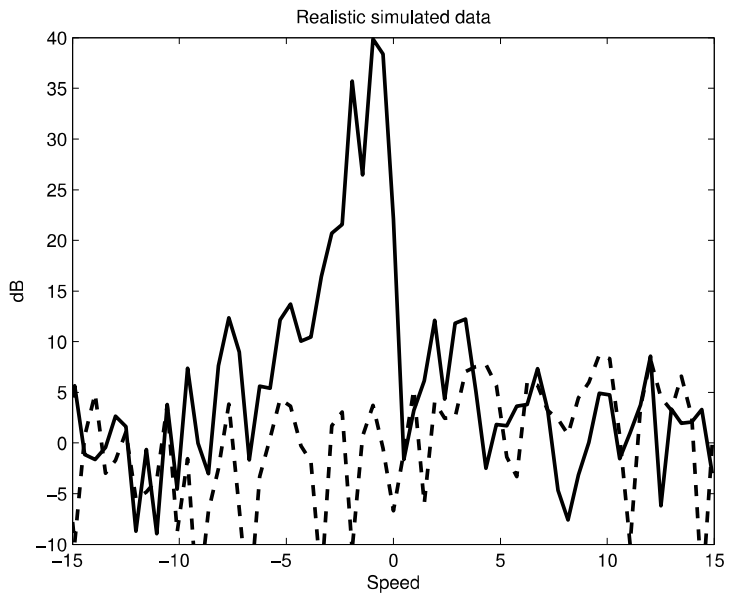

Fig. 3. Comparison between sum channel (bold curve, negative speeds), deterministic (bold, positive speeds) and STAP (dash) on realistic data

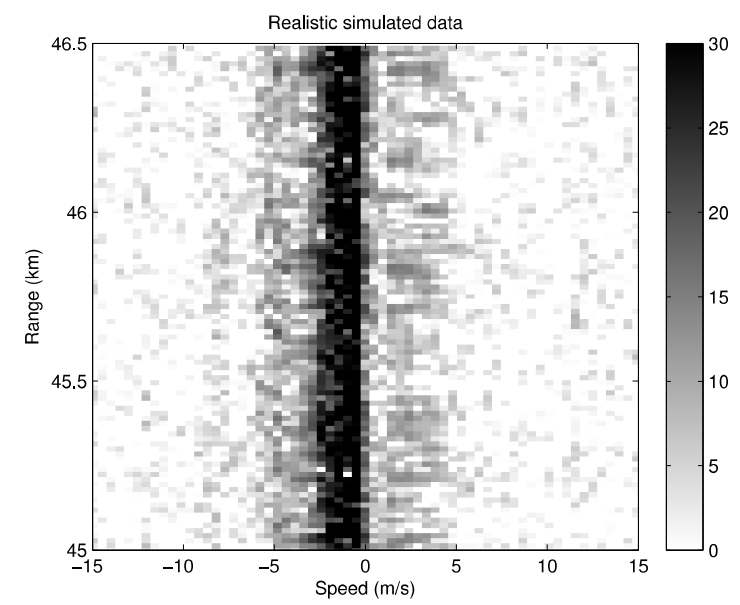

Fig. 4. Range-Doppler map of the non-adaptive processing (postitive speeds) and the sum channel (negative speeds) on realistic data

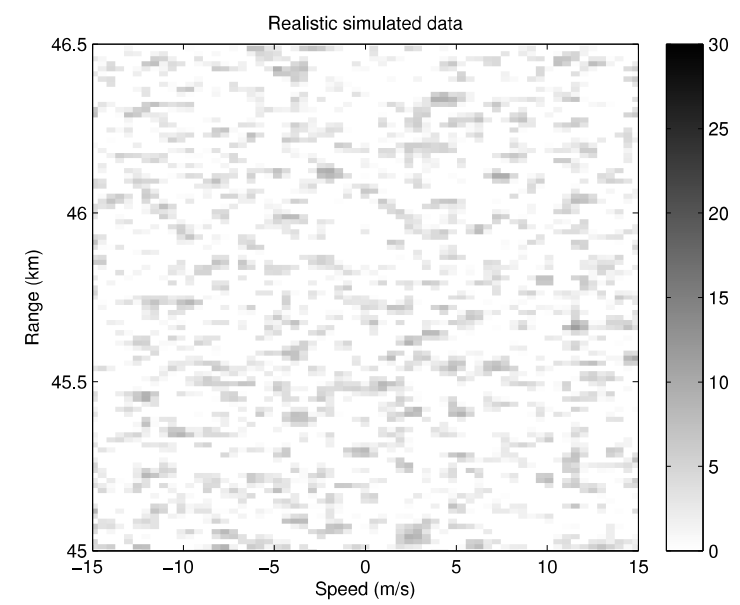

Fig. 5. Range-Doppler map of the adaptive processing on realistic data 


\section{DETERMINISTIC-AIDED STAP}

In GMTI operation, there are two main concerns about heterogeneous environments: clutter heterogeneity (land relief, urban environments) and high density target area (roads, highways...). In many cases, few training data are available and the use of single data set methods is a very helpful alternative (see Section II.A and Section III.B). To overcome the problem of these methods pointed out in Section III.C, we propose a new method that includes some aspects of the non-adaptive processing. We saw in (14) that the term $\mathbf{g g}^{\mathbf{H}}$ in (8) removes the interest signal (if any) but also a small part of the clutter. The idea here is to try to re-add this clutter into the covariance matrix. The covariance matrix is then:

$$
\mathbf{T}=\mathbf{R}-\mathbf{g g}^{\mathbf{H}}+\mathbf{g}_{\mathbf{c}} \mathbf{g}_{\mathbf{c}}^{\mathbf{H}}
$$

where $\mathbf{g}_{\mathbf{c}}$ is the projection of $\mathbf{g}$ on the clutter steering vector $\mathbf{s}_{\mathbf{c}}(\theta(f), f)$ :

$$
\mathrm{g}_{\mathrm{c}}=\mathbf{P}_{\mathrm{c}} \mathrm{g}
$$

with

$$
\mathbf{P}_{c}=\frac{\mathbf{s}_{\mathbf{c}}(\theta(f), f) \mathbf{s}_{\mathbf{c}}^{H}(\theta(f), f)}{\mathbf{s}_{\mathbf{c}}^{H}(\theta(f), f) \mathbf{s}_{\mathbf{c}}(\theta(f), f)}
$$

We can demonstrate that the covariance matrix $\mathbf{Q}$ of (14) can now be written as follows:

$$
\mathbf{T}=\frac{\mathbf{N N}^{\mathbf{H}}}{K_{t}}-\frac{\mathbf{N} \mathbf{s}_{\mathbf{t}}^{*} \mathbf{s}_{\mathbf{t}}^{\mathbf{T}} \mathbf{N}^{\mathbf{H}}}{K_{t}^{2}}+\frac{\mathbf{P}_{\mathbf{c}} \mathbf{X} \mathbf{s}_{\mathbf{t}}^{*} \mathbf{s}_{\mathbf{t}}^{\mathbf{T}} \mathbf{X}^{\mathbf{H}} \mathbf{P}_{\mathbf{c}}^{\mathbf{H}}}{K_{t}^{2}}
$$

If the clutter follows the theoretical Doppler-angle relation of (15), then the projection of the signal on the angle-Doppler steering vector will be close to the clutter signal that has been removed from the matrix $\left(\mathbf{P}_{\mathbf{c}} \mathbf{X} \approx \mathbf{N}\right)$, and the covariance matrix $\mathbf{T}$ will be close to:

$$
\mathbf{T} \approx \frac{\mathbf{N N}^{\mathbf{H}}}{K_{t}}
$$

Note that we don't need to set an arbitrary clutter power value because the energy of the clutter is included in $\mathbf{g}_{\mathbf{c}}$ (cf. (19)). In the case of Stop-Band APES, where the signal notch is wider, we use an extended projector $\mathbf{P}_{\mathbf{c}}$ :

with

$$
\mathbf{P}_{c}=\frac{\mathbf{S}_{\mathbf{c}} \mathbf{S}_{\mathbf{c}}^{\mathbf{H}}}{\mathbf{S}_{\mathbf{c}}^{\mathbf{H}} \mathbf{S}_{\mathbf{c}}}
$$

$$
\mathbf{S}_{\mathbf{c}}=\left[\mathbf{s}_{\mathbf{c}}\left(\theta, f-\frac{\Delta f}{2}\right) \mathbf{s}_{\mathbf{c}}(\theta, f) \mathbf{s}_{\mathbf{c}}\left(\theta, f+\frac{\Delta f}{2}\right)\right]
$$

\section{RESUlts}

We test the GMTI deterministic-aided STAP described in the previous section on real airborne data. These data were obtained using the ONERA RAMSES radar system [14], which is a 4 channels ULA antenna. The aircraft speed was $V_{a}=$ $85 \mathrm{~m} . \mathrm{s}^{-1}$, pulse repetition frequency is $\mathrm{PRF}=1.5625 \mathrm{kHz}$, the number of range gates is 300 , the number of time taps used to form the space-time data is Ktaps $=7$ and the total number of time snapshots (radar pulses) is 64. Three targets are present in the scene:

\begin{tabular}{|c|c|c|c|}
\hline & target 1 & target 2 & target 3 \\
\hline Speed (m/s) & 3.0 & 5.30 & 5.85 \\
\hline Range (number) & 214 & 138 & 149 \\
\hline
\end{tabular}

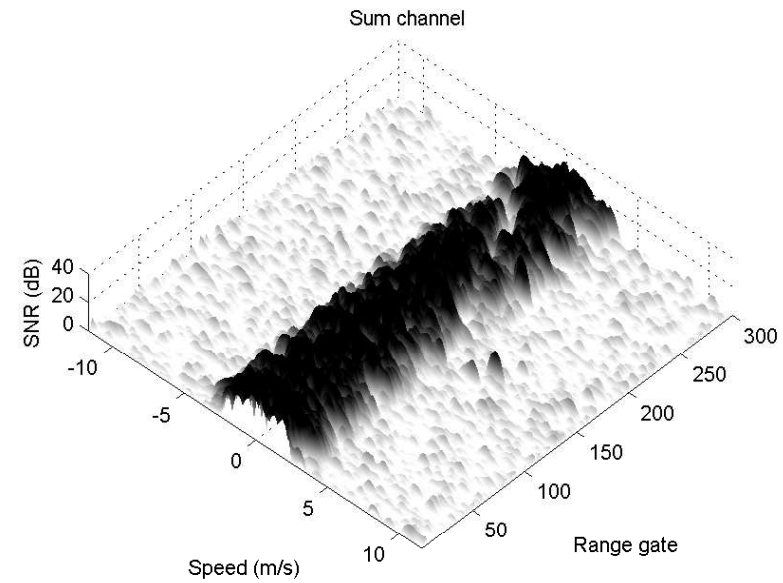

Fig. 6. Range-Doppler map showing the sum channel of the RAMSES data

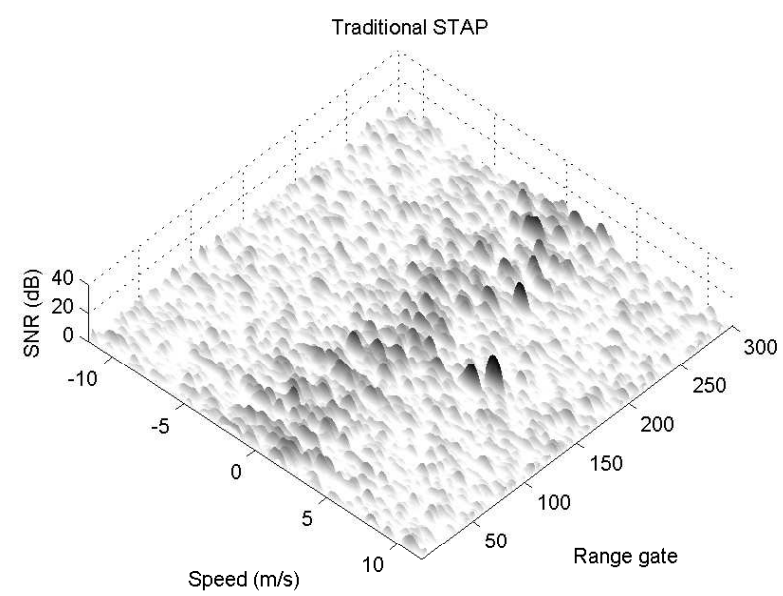

Fig. 7. Range-Doppler map on RAMSES data showing the performance of classical STAP processing

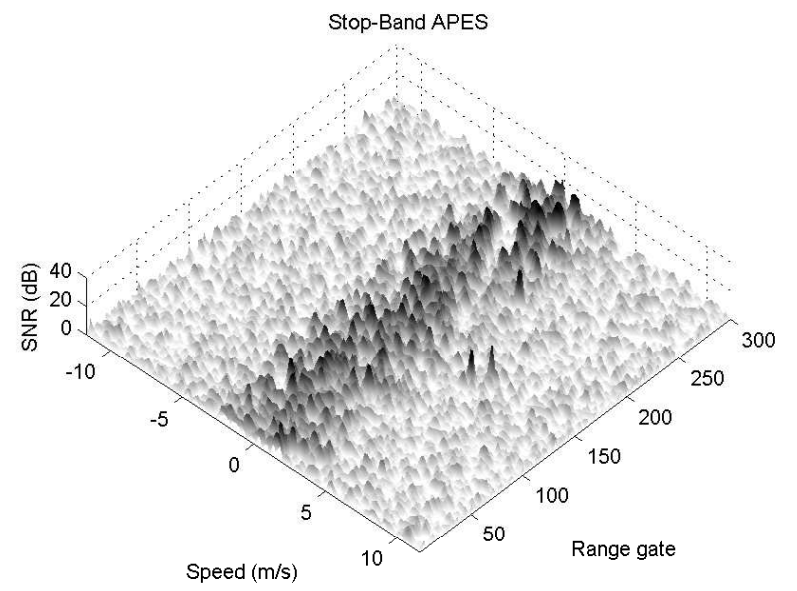

Fig. 8. Range-Doppler map on RAMSES data showing the performance of the Stop-Band detector 


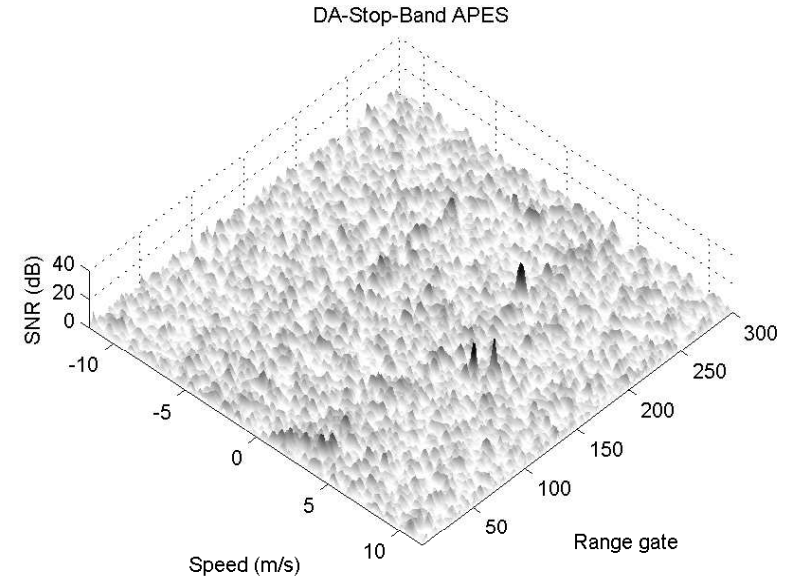

Fig. 9. Range-Doppler map on RAMSES data showing the performance of the deterministi-aidedc Stop-Band detector

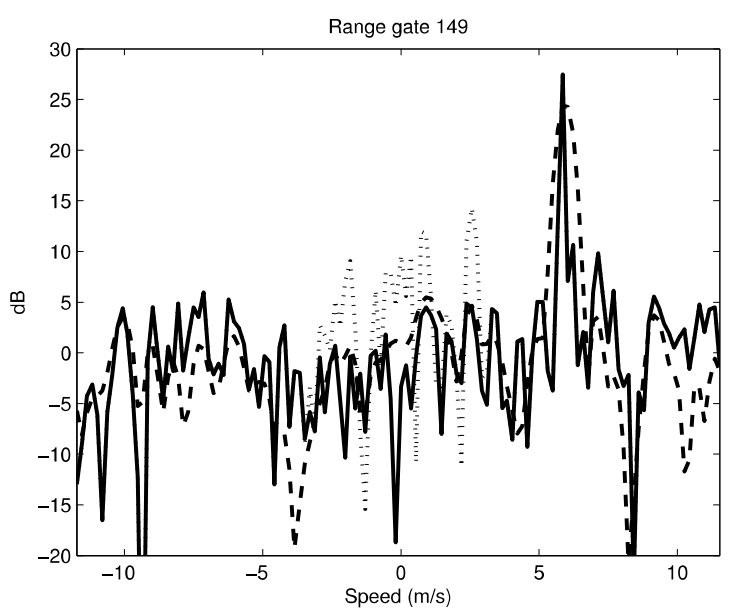

Fig. 10. Comparison of classical STAP (dash curve) Stop-Band STAP (dot curve) and deterministic-aided Stop-Band (solid curve) on RAMSES data for range gate number 149

The Doppler-range of the sum channel (Fig. 6) cleary emphasizes the heterogeneous clutter. The next figures present the results for classical STAP (estimation on 10 range gates with 2 guardcells), MLED STAP, Stop-Band STAP and deterministicaided Stop-Band STAP (estimation on 3 range gates with no guard-cells for all these processings). No oversampling is used for the STAP processors (although a $2 \mathrm{x}$ zero-padding is needed to access the data of the half-resolution Doppler cells in the case of Stop-Band) except for the MLED detector, which use a $4 x$-oversampling.

The classical STAP processing fails to correctly remove the heterogenous clutter (Fig. 7). The MLED STAP which signal notch is very sharp also fails to completely remove the clutter. Due to its property of high resolution, the target Doppler extent is very thin and it is difficult to distinguish the targets on the range-Doppler map (Fig. ??). As predicted, the Stop-Bland STAP processing let even more clutter to be present as shown on Fig. 8, whereas the deterministic-aided Stop-Band (DAStop-Band) effectively cancels the clutter (Fig. 9). This is done without any attenuation on target 1 which lies in the clutter.
Figure 10 points out the increased clutter attenuation if DAStop-Band over classical Stop-Band for range gate number 149 , where target 3 is present.

\section{CONCLUSION}

In this paper, we propose two deterministic-aided algorithms both based on the APES method. The first algorithm which relies on the deterministic Doppler-angle relation of the clutter is particularly adapted for GMTI detectors. The results on real data show that it outperforms both classical STAP and APES-based algorithms. The second algorithm, which aims to remove the continuous component of the interference, is on the other hand well adapted to air-to-air modes. In this case, the continuous interference is the main lobe clutter. On realistic simulated data, it totally cancels the main lobe clutter, whereas classical STAP and traditional APES-based algorithms fail, causing many false alarms.

\section{REFERENCES}

[1] W. Melvin, "A STAP overview," IEEE Aerospace And Electronic Systems Magazine, vol. 19, no. 1, pp. 19-35, 2004.

[2] R. Klemm, Principles of Space-time adaptive processing. The Institution of Electrical Engineers (IEE), 2002.

[3] E. Aboutanios and B. Mulgrew, "Evaluation of the single and two data set STAP detection algorithms using measured data," 2007 IEEE International Geoscience and Remote Sensing Symposium, pp. 494-498, 2007.

[4] P. Wang, H. Li, and B. Himed, "A new parametric GLRT for multichannel adaptive signal detection," Signal Processing, IEEE Transactions on, vol. 58, no. 1, pp. $317-325$, jan. 2010.

[5] E. Aboutanios and B. Mulgrew, "Hybrid detection approach for STAP in heterogeneous clutter," Aerospace and Electronic Systems, IEEE Transactions on, vol. 46, no. 3, pp. $1021-1033$, july 2010.

[6] M. Zatman, "Properties of hung-turner projections and their relationship to the eigencanceller," in Signals, Systems and Computers, 1996. Conference Record of the 30th Asilomar Conference on.

[7] E. Aboutanios and B. Mulgrew, "A STAP algorithm for radar target detection in heterogeneous environments," in Statistical Signal Processing, 2005 IEEE/SP 13th Workshop on.

[8] L. H. Stoica P. and J. Li, "A new derivation of the apes filter," IEEE Signal Processing Letters, pp. 205-206, 1999.

[9] L. Brennan and I. Reed, "Theory of adaptive radar," IEEE Transactions On Aerospace And Electronic Systems, vol. AES-9, no. 2, pp. 237-252, 1973.

[10] Y. Kim, S. Pillai, and J. Guerci, "Optimal loading factor for minimal sample support space-time adaptive radar," in Acoustics, Speech and Signal Processing, 1998. Proceedings of the 1998 IEEE International Conference on, vol. 4, may 1998, pp. $2505-2508$ vol.4.

[11] J.-F. Degurse, S. Marcos, and L. Savy, "Subspace-based and single dataset methods for STAP in heterogeneous environments," in Proceedings of the IET RADAR 2012 Conference, Glasgow, United Kingdom, Oct. 2012, pp. 1-6. [Online]. Available: http: //hal-supelec.archives-ouvertes.fr/hal-00776374

[12] J.-F. Degurse, L. Savy, R. Perenon, and S. Marcos, "An extended formulation of the maximum likelihood estimation algorithm. application to space-time adaptive processing," in Radar Symposium (IRS), 2011 Proceedings International, sept. 2011, pp. $763-768$.

[13] L. Savy and J.-F. Degurse, "Stop-band apes: Traitements adaptatifs en environnements heterogenes," Revue Traitement du Signal, vol. 28, no. 1/2, pp. 231-256, december 2011.

[14] P. Dubois-Fernandez, O. Ruault du Plessis, D. Le Coz, J. Dupas, B. Vaizan, X. Dupuis, H. Cantalloube, C. Coulombeix, C. TitinSchnaider, P. Dreuillet, J. Boutry, J. Canny, L. Kaisersmertz, J. Peyret, P. Martineau, M. Chanteclerc, L. Pastore, and J. Bruyant, "The ONERA RAMSES SAR system," in Geoscience and Remote Sensing Symposium, 2002. IGARSS '02. 2002 IEEE International, vol. 3, 2002, pp. 1723-1725 vol.3. 